\title{
CMEARTICLE
}

\section{Clinics in diagnostic imaging (155)}

May San Mak ${ }^{1}$, MBChB, FRCR, Ching Ching Ong ${ }^{1}$, MBBS, FRCR, Edgar Lik Wui Tay ${ }^{2}$, MBBS, MRCP, Lynette Li San $\underline{\text { TeO }}^{1}, \mathrm{MBChB}, \mathrm{FRCR}$

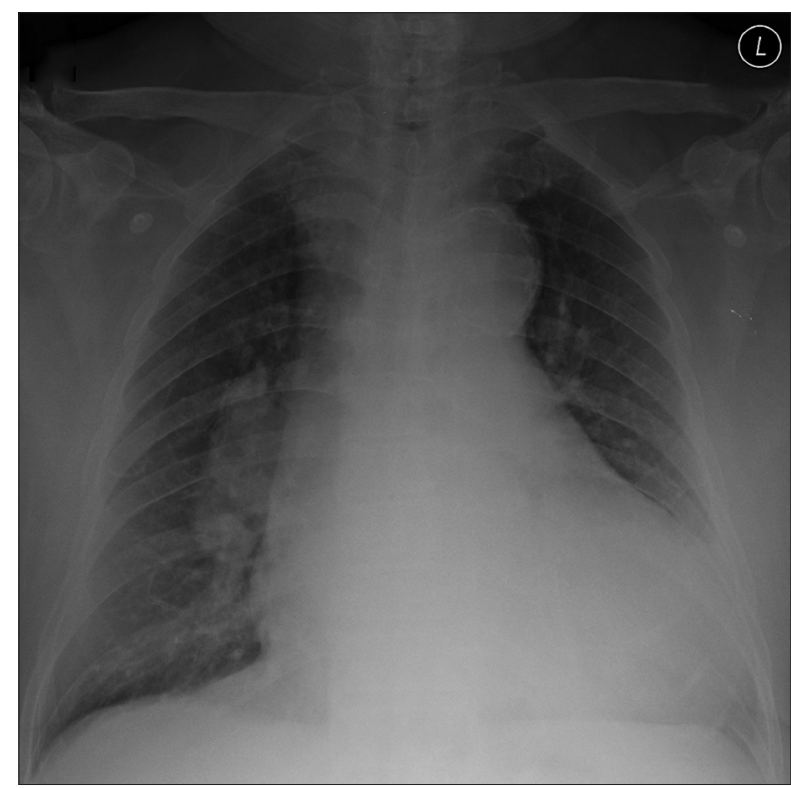

Fig. 1 Chest radiograph of the patient.
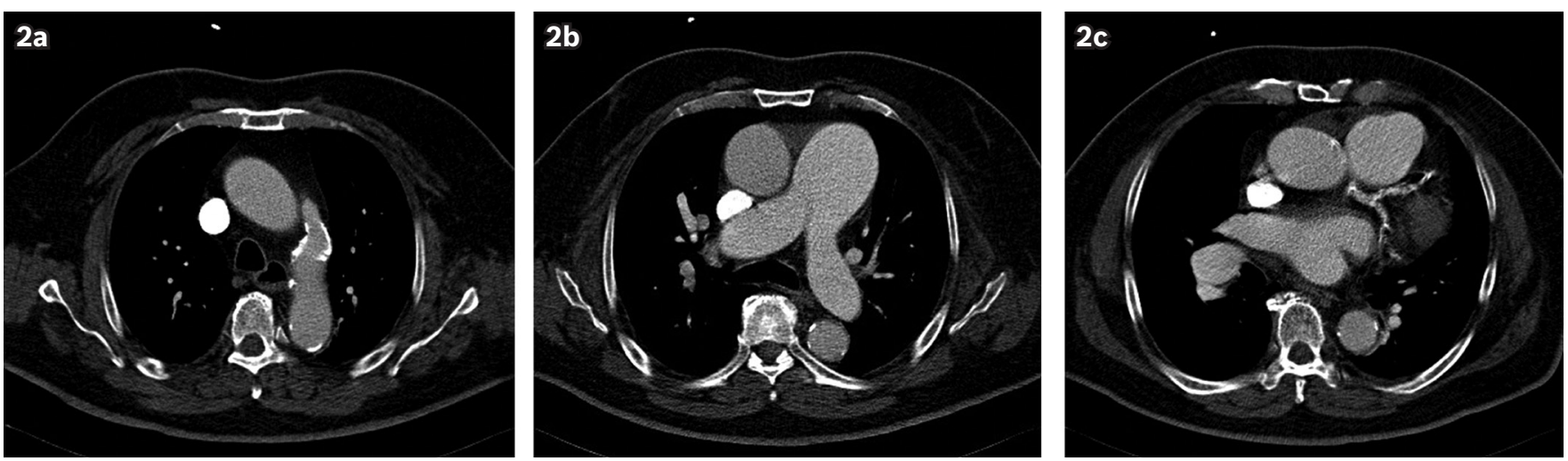

Fig. $2(\mathrm{a}-\mathrm{c})$ Selected contrast-enhanced axial CT images.

\section{CLINICAL PRESENTATION}

An obese 70-year-old man presented with an acute onset of chest pain and dyspnoea, as well as longstanding lower limb swelling. The patient had a background history of essential hypertension, hyperlipidaemia and ischaemic heart disease, and had undergone percutaneous coronary intervention in an overseas institution three years earlier. Otherwise, he was in a good general state of health prior to this presentation. Physical examination revealed an incidental continuous cardiac murmur, best heard at the left upper sternal border.

Blood results of the patient were as follows: D-dimer 347.6 (normal range $[\mathrm{NR}]<230.0) \mathrm{ng} / \mathrm{mL}$; creatine kinase 223 (NR
30-350) U/L; creatine kinase MB 2.7 (NR 1.0-6.0) $\mu \mathrm{g} / \mathrm{L}$; and troponin I 0.059 (NR 0.000-0.039) $\mu \mathrm{g} / \mathrm{L}$. Serial cardiac enzymes showed a rising trend (i.e. creatine kinase $262 \mathrm{U} / \mathrm{L}$, creatine kinase MB $5.7 \mu \mathrm{g} / \mathrm{L}$ and troponin I $0.123 \mu \mathrm{g} / \mathrm{L}$ ).

Transthoracic echocardiography found dilated pulmonary arteries with an estimated pulmonary arterial systolic pressure (PASP) of $51 \mathrm{mmHg}$, a reading higher than that expected for ischaemic cardiomyopathy or myocardial infarction. Chest radiography was performed (Fig. 1). Triple-rule-out (TRO) cardiacgated multidetector computed tomography (MDCT) (Fig. 2) was also performed to further investigate his symptoms and the disproportionate elevation of PASP. What do these images show?

${ }^{1}$ Department of Diagnostic Imaging, ${ }^{2}$ Department of Cardiology, National University Hospital, Singapore

Correspondence: Dr Lynette Teo, Senior Consultant, Department of Diagnostic Imaging, National University Hospital, 5 Lower Kent Ridge Road, Singapore 119074. lynette_Is_teo@nuhs.edu.sg 


\section{IMAGE INTERPRETATION}

Conventional chest radiography (Fig. 1) shows cardiomegaly with dilatation of all four cardiac chambers. The pulmonary arteries are dilated, with the right interlobar pulmonary artery measuring at least $29 \mathrm{~mm}$ in calibre. The upper limit of normal for the transverse diameter of the proximal right interlobar artery, as measured on chest radiography at a level immediately lateral to the proximal portion of the bronchus intermedius, is $17 \mathrm{~mm}$ in men and $15 \mathrm{~mm}$ in women. ${ }^{(1)}$ The findings are in keeping with pulmonary arterial hypertension. There is no peripheral pruning.

Contrast-enhanced axial MDCT images show a tubular structure connecting the proximal part of the descending thoracic aorta to the roof of the proximal left pulmonary artery, which is consistent with a patent ductus arteriosus (PDA). The PDA is funnel-shaped in morphology. There is mural calcification of the PDA predominantly along the aortic end of the PDA. Mural calcification of the aortic arch is also seen (Fig. 2a). There is pulmonary arterial hypertension, as evidenced by the dilatation of the pulmonary arteries. The calibre of the main pulmonary artery is larger than that of the ascending aorta, measuring $56 \mathrm{~mm}$ and $45 \mathrm{~mm}$, respectively (Fig. 2b). There is also extensive calcification of the left main coronary artery, left anterior descending artery and left circumflex artery (Fig. 2c).

\section{DIAGNOSIS}

Incidental PDA with secondary pulmonary arterial hypertension.

\section{CLINICAL COURSE}

Cardiac catheterisation confirmed the presence of a large PDA, moderate pulmonary arterial hypertension (mean pulmonary arterial pressure was $44 \mathrm{mmHg}$ ) and double-vessel coronary artery disease. Stent thrombosis of the high mid-left anterior descending artery and severe in-stent restenosis of the mid-right coronary artery were diagnosed. The multidisciplinary team decided that a surgical option was preferred over a transcatheter option in view of the coexisting severe coronary artery disease and PDA. The patient then underwent coronary artery bypass surgery and concomitant GoreTex patch closure of the PDA on the pulmonary side via a main pulmonary arteriotomy (Fig. 3). He remained well over the subsequent two-year follow-up period.

\section{DISCUSSION}

The ductus arteriosus is a vital fetal vascular structure that connects the proximal descending thoracic aorta to the roof of the main pulmonary artery or proximal left pulmonary artery. ${ }^{(2)}$ The ductus arteriosus is essential for diverting right ventricular output away from the high-resistance fetal pulmonary circulation into the descending thoracic aorta and systemic arterial circulation. ${ }^{(3,4)}$ Functional closure from vasoconstriction, with contraction of the medial smooth muscle in the ductus, occurs within 24-48 hours after delivery. Within the next 2-3 weeks, permanent structural closure occurs as a result of fibrosis. ${ }^{(2,3)}$ The resulting fibrous band persists as the ligamentum arteriosum. After the first three months of life, persistence of ductal

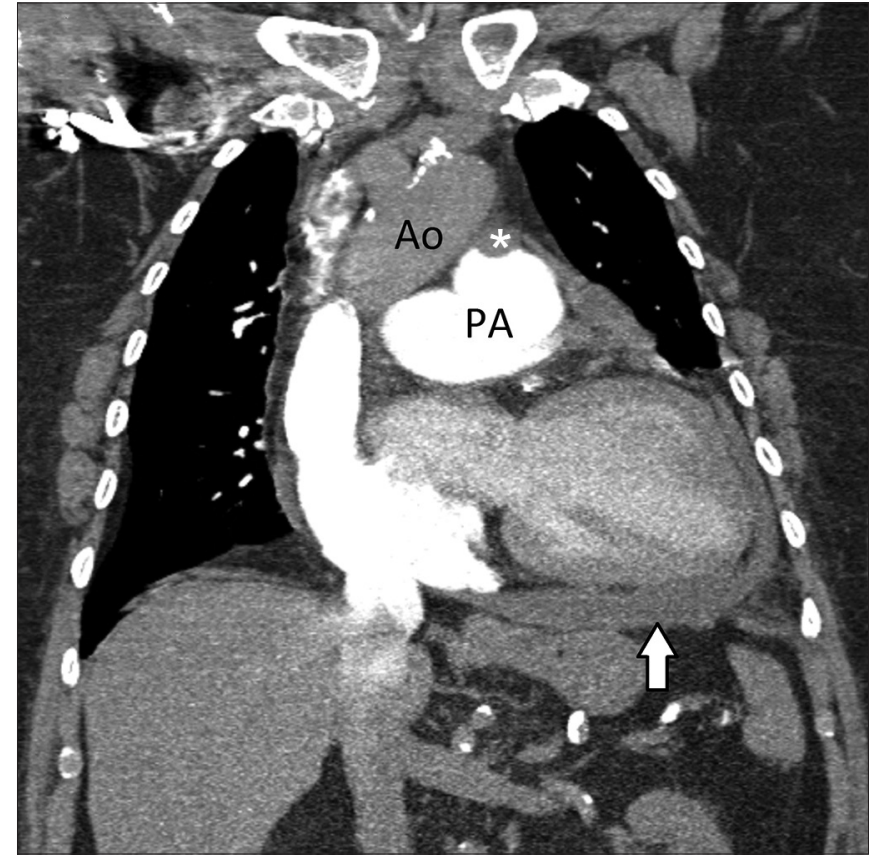

Fig. 3 Selected coronal image from a CT pulmonary angiogram after surgical closure of the patent ductus arteriosus. Patch closure was performed on the pulmonary artery side, as evidenced by the concave indentation on the roof of the proximal left pulmonary artery (asterisk). There is no mixing of the unenhanced blood in the thoracic aorta (Ao) with the densely opacified central pulmonary arteries (PA). A small amount of postoperative haemopericardium is also present (arrow)

patency with continuous flow from the descending aorta to the central pulmonary arteries, resulting in a left-to-right shunt, is considered pathological. ${ }^{(2)}$

Isolated PDA is the third most common congenital heart anomaly, and is estimated to account for approximately $10 \%-12 \%$ of such cases. ${ }^{(3,5,6)}$ Data presented by Hoffman and Kaplan, which was taken from 44 published studies on the incidence of congenital heart disease, shows that ventricular septal defect was by far the most common lesion at birth, followed by atrial septal defect, PDA and pulmonic stenosis. ${ }^{(7)}$ There is a higher incidence of PDA among preterm infants, and in most studies, the male to female ratio has been reported to be $1: 2 .^{(2)}$ While PDA is usually diagnosed early in life, subclinical PDA is a rare entity in the adult and elderly population, ${ }^{(8,9)}$ traditionally investigated and diagnosed only after the patient becomes symptomatic.(5) Occasionally, silent PDA may be discovered incidentally on MDCT performed for other purposes. ${ }^{(5)}$ With the widespread use of MDCT today, it is important that this condition is recognised, as it can be easily missed unless special attention is directed to this specific anatomic region. ${ }^{(5)}$

The haemodynamic consequences and clinical manifestations of a PDA are determined by the magnitude of shunting, which depends on the resistance of the ductus and pulmonary vasculature status. ${ }^{(2,5)}$ The flow resistance of the ductus depends on the overall shape and size of the ductus, as well as its elasticity. The pressure gradient between the aorta and pulmonary artery results in left-to-right shunting through the ductus, resulting in pulmonary overcirculation and left heart volume overload. ${ }^{(2)}$ Thus, the natural history of a haemodynamically significant PDA has typically been associated with cardiovascular derangements, 

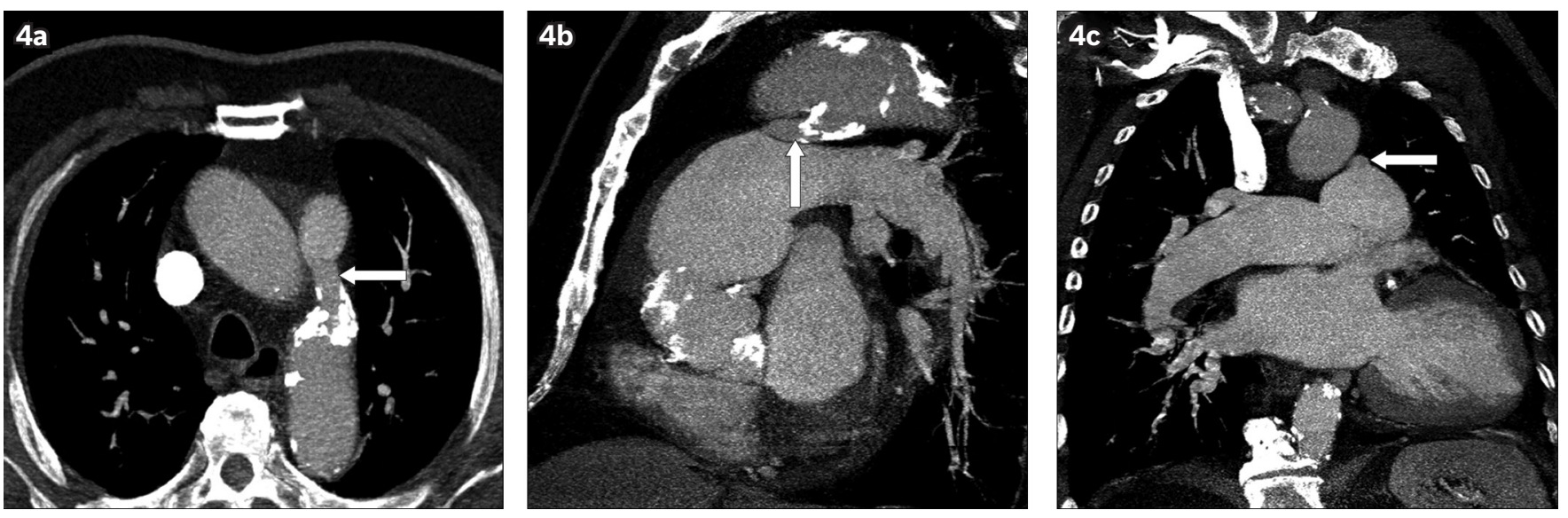

Fig. 4 Multiplanar reformation CT images confirm the presence of a PDA (arrows). (a) Axial, (b) sagittal and (c) coronal oblique images show a clearer visualisation of the ductal morphology and the extent of calcification, and allow for precise measurement of ductal dimensions.

including congestive heart failure, arrhythmias and progressively severe pulmonary hypertension. ${ }^{(6)}$ With longstanding exposure to increased pressure, the pulmonary vasculature undergoes morphological changes, resulting in increased vascular resistance. If the pulmonary vascular resistance exceeds that of the systemic circulation, the flow within the duct reverses to right-to-left. Eisenmenger's syndrome is an irreversible complication that occurs in untreated congenital heart anomalies where a left-toright shunt is converted into a right-to-left shunt secondary to longstanding elevated pulmonary arterial pressure and associated pulmonary vascular disease.

A minority of PDAs may be haemodynamically well tolerated and remain subclinical into adulthood.(4) These usually involve relatively small left-to-right shunts that do not significantly overload the pulmonary vascular tree; hence, this subset of patients survive longer..$^{(4,6,10)}$ Fisher et al suggested that some patients may tolerate very high pulmonary arterial pressure for long periods without deterioration of their clinical status. ${ }^{(9)}$ However, unrepaired PDA in these asymptomatic patients may eventually become clinically significant, particularly in conjunction with an acquired physiological insult from recurrent pneumonia, development of chronic obstructive pulmonary disease, ischaemic heart disease or calcific aortic stenosis. ${ }^{(2,3)}$ In addition, there is an associated risk of infective endarteritis, even in small PDAs, estimated to be up to $0.45 \%$ per year after the second decade of life. ${ }^{(4)}$ Indeed, infective endocarditis was a common fatal complication of PDA before the introduction of antibiotic therapy. ${ }^{(5)}$

The most specific sign of a PDA on chest radiography is two parallel lines due to mural calcification of the ductus, known as the 'railroad track' sign. However, this is rarely seen, and even if present, it is often appreciated only in retrospect. More commonly, chest radiographs will manifest complications of PDA, such as heart failure and pulmonary arterial hypertension, showing cardiomegaly with or without signs of pulmonary oedema and enlargement of the pulmonary arteries. Unfortunately, these findings are nonspecific, as any cause of a left-to-right shunt can produce similar features. Certainly, if the amount of ductal shunting is small, the chest radiograph may be completely normal. ${ }^{(2)}$
Our patient presented with acute chest pain and had elevated cardiac enzymes suspicious of acute coronary syndrome. However, because of the disproportionately elevated PASP, other causes of pulmonary arterial hypertension had to be ruled out, and thus a TRO MDCT was performed. TRO MDCT is cardiac-gated and optimised for evaluation of the coronary arteries, aorta and pulmonary arteries, as well as the adjacent intrathoracic structures. TRO MDCT provides the additional benefit of making noncoronary diagnoses. ${ }^{(11)} \mathrm{A}$ study by Takakuwa and Halpern, conducted in patients presenting to the emergency department with acute chest pain, found that TRO MDCT identified $11 \%$ of noncoronary diagnoses that explained the presenting symptom, and $14 \%$ of clinically important noncoronary diagnoses that did not explain the presenting symptom. ${ }^{(12)}$ Therefore, TRO MDCT is considered most appropriate, time-efficient and cost-effective when acute coronary syndrome, along with other diagnoses such as pulmonary embolism, acute aortic syndrome or nonvascular disease in the thorax, is suspected, eliminating the need for separate dedicated studies for these acute chest conditions. ${ }^{(11)}$

MDCT, together with techniques such as multiplanar $\mathrm{CT}$ reformation and three-dimensional volume-rendered $\mathrm{CT}$ reconstruction, can help to establish the diagnosis of PDA. As PDA anatomy varies considerably from patient to patient, ${ }^{(4)}$ the information provided by MDCT is crucial to the planning of optimal treatment.

Multiplanar CT reformation provides precise information on the size and morphology of a PDA, as well as the presence and extent of calcification (Fig. 4). ${ }^{(5,8)}$ Sizing and morphologic classification of PDA, which traditionally required invasive technique, can now be accurately done with multiplanar CT reformation. ${ }^{(8)}$ Size remains an important factor in the evaluation of adults with PDA, as size criteria determine whether transcatheter closure may be performed, and if suitability is established, they also determine the selection of correct closure device type and size. Detailed morphologic information on the duct provided by multiplanar CT reformation helps to identify the subset of patients for surgery, typically involving large or unfavourable PDA geometry. ${ }^{(2,5)}$ The extent of mural calcification of the ductus and adjacent aorta is particularly important if 


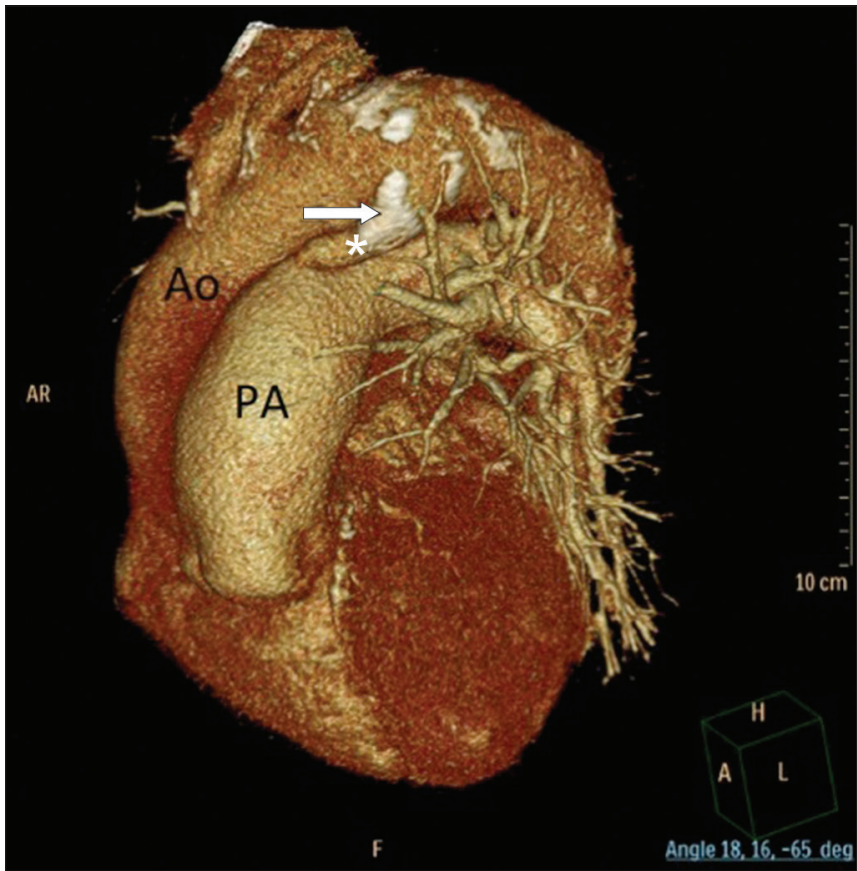

Fig. 5 Three-dimensional volume-rendered CT model shows the paten ductus arteriosus (PDA) (asterisk) morphology, and its spatial relationship to the aorta (Ao) and pulmonary artery (PA). Mural calcification is also seen in the aortic side of the PDA (arrow).

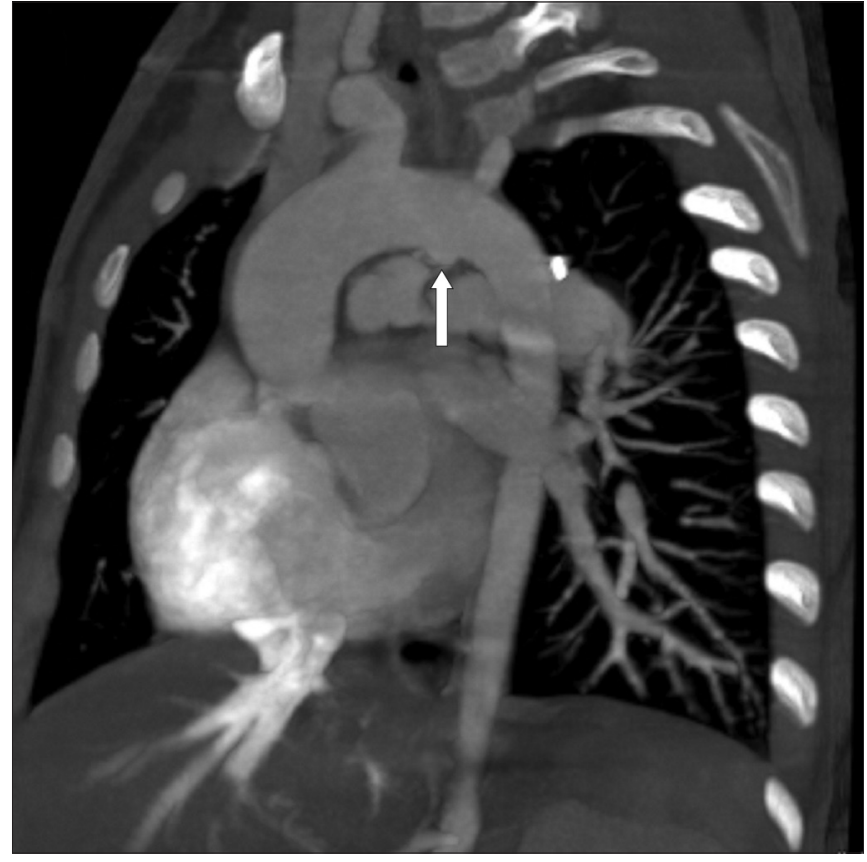

Fig. 6 Sagittal oblique CT reformation (candy cane aorta view) image of an 18-month-old patient shows a ductus diverticulum (arrow), which is easily differentiated from a patent ductus arteriosus on multiplanar CT reformation, as no connection to the central pulmonary arteries is demonstrated.
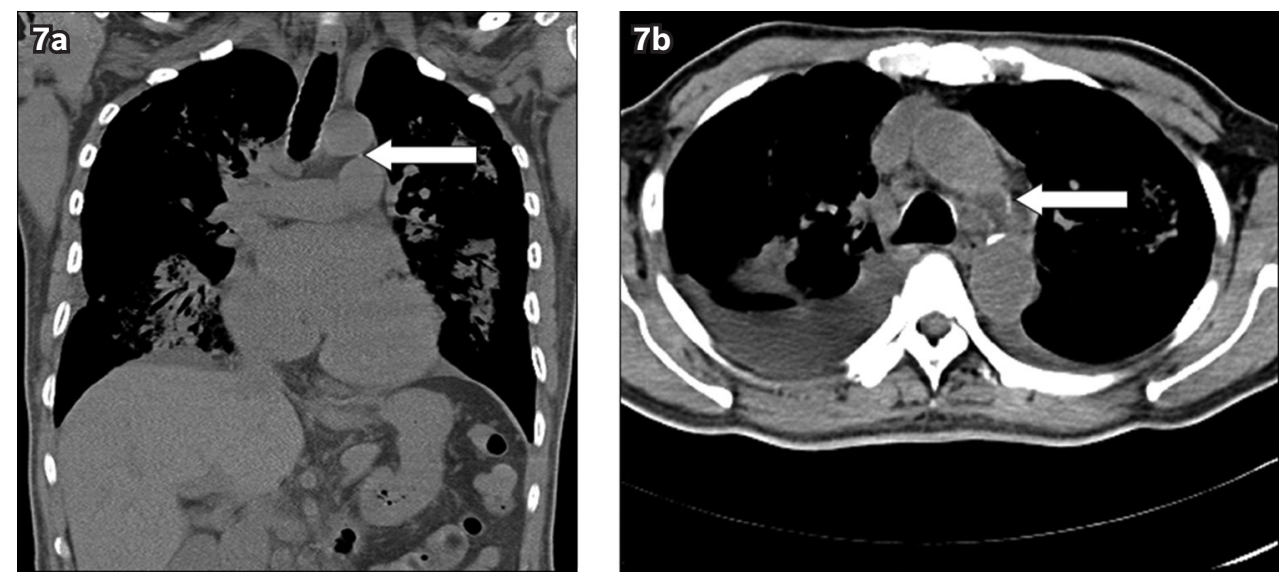

Fig. 7 Unenhanced (a) coronal and (b) axial CT images of 65-year-old patient show a linear calcified band in the aortopulmonary window, in keeping with a calcified ligamentum arteriosum (arrows). Sometimes, this is mistaken for a migrated and impacted foreign body from the proximal thoracic oesophagus.

surgical therapy is considered (Fig. 2a). ${ }^{(8,13)}$ Wall-calcified ductus are common in adult PDAs, with a reported incidence of up to $14 \%{ }^{(9)}$ Calcification and atherosclerotic lesions make the ductus fragile and friable, increasing the technical difficulty of the surgical procedure. . $^{(3,10,14)}$

Three-dimensional volume-rendered $\mathrm{CT}$ reconstruction allows for clear visualisation of the ductal morphology and demonstrates its spatial relationship to adjacent structures, including the aorta and pulmonary artery (Fig. 5). ${ }^{(5,8)}$ Additionally, knowledge of the anatomy of the PDA and its relationship to the trachea is potentially useful information to have before performing transcatheter closure, as the tracheal shadow provides a fixed landmark that helps to guide device positioning. ${ }^{(2,8)}$

MDCT helps to differentiate mimickers of PDA such as a small diverticulum at the site of the ductus, which is a remnant of the ductus arteriosus (Fig. 6). ${ }^{(5)}$ While the diverticulum may be mistaken for a PDA on axial images, it is easily differentiated using multiplanar reformation. Interestingly, the ligamentum arteriosum can sometimes be seen as a calcified linear band on routine MDCT, and may sometimes be misinterpreted as a migrated and impacted foreign body from the proximal thoracic oesophagus (Fig. 7).

Ductus closure is indicated in adults with medium- and large-sized PDAs with significant left-to-right shunting, even in asymptomatic cases, to minimise the risk of complications in the future. (2) Elimination of the shunt reduces volume overload of the pulmonary vasculature and left ventricle, preventing pulmonary vascular obstructive disease and congestive heart failure. $^{(3)}$ Indications for closure of small PDAs, including those that are tiny and incidentally discovered, are less certain. ${ }^{(2,3)}$ 


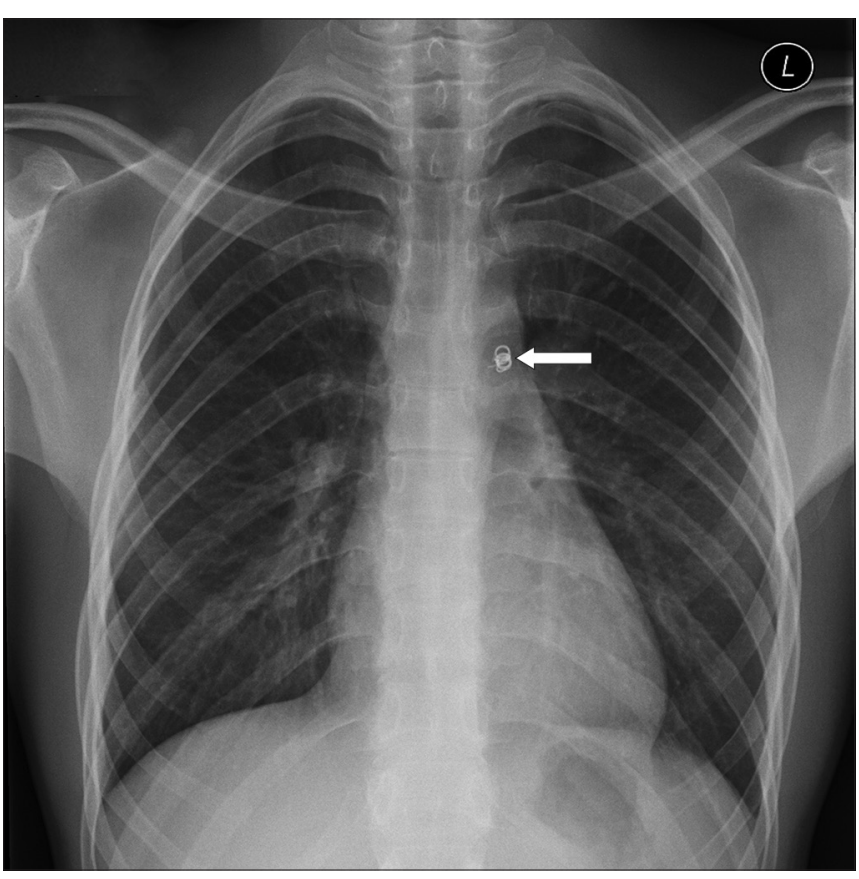

Fig. 8 Chest radiograph of a 20-year-old patient with a transcatheter PDA occlusion device (arrow).

However, due to the risk of endarteritis and as current closure methods are generally safe and effective with minimal morbidity, several authors recommend elective closure of any PDA, unless irreversible severe pulmonary hypertension is present. ${ }^{(2-4)}$ Neither surgical nor transcatheter closure of a PDA is recommended in cases of nonreversible pulmonary hypertension and Eisenmenger's syndrome, as its closure is accompanied by high mortality and may not help improve symptoms or prevent cor pulmonale. ${ }^{(14)}$

Since the first successful surgical ligation reported in 1939, many different surgical and catheter-based approaches have been developed for permanent closure of isolated PDAs. ${ }^{(2,4)}$ For adults, nonsurgical transcatheter PDA closure is the treatment of choice whenever possible, as it is less invasive, causes less trauma to patients and costs less (Fig. 8). ${ }^{(2-4)}$ The results of transcatheter occlusion of PDA have been excellent. ${ }^{(2,4)}$ However, for adults with large or unfavourably shaped ductus, surgical ligation is an effective alternative. ${ }^{(3)}$ Additionally, surgery may be a better closure option in cases of PDA with coexistent disease, such as in our patient described above. ${ }^{(8)}$ Although generally associated with greater morbidity than transcatheter methods, surgical closure is a generally safe and effective procedure. ${ }^{(2)}$

In conclusion, isolated subclinical PDA in adults is an uncommon entity, although it may be identified incidentally on CT performed for other purposes. With the widespread use of CT, recognition of this condition is important. There is also the added advantage of concomitant noninvasive assessment of the ductal and regional anatomy for planning of optimal treatment. The aforementioned case illustrates an incidental PDA co-presenting with acute coronary syndrome diagnosed on TRO cardiac-gated MDCT. The patient underwent surgical patch closure of the
PDA with concurrent coronary artery bypass surgery and had an uneventful recovery.

ABSTRACT We report the case of a 70-year-old man with an asymptomatic large patent ductus arteriosus (PDA) incidentally detected on triple-rule-out computed tomography (CT). CT clearly demonstrated a vascular structure connecting the descending thoracic aorta to the roof of the proximal left pulmonary artery, consistent with a PDA. Secondary pulmonary arterial hypertension was also evident on CT. The patient was eventually diagnosed with acute coronary syndrome and was successfully treated with coronary artery bypass graft surgery and concomitant patch closure of the PDA. This article aims to outline the imaging features of PDA and highlight the information provided by $\mathrm{CT}$, which is crucial to treatment planning. The pathophysiology, clinical manifestations and closure options of PDA are also briefly discussed.

Keywords: congenital heart disease, patent ductus arteriosus, pulmonary arterial hypertension, triple-rule-out computed tomography

\section{REFERENCES}

1. Miro S, Klein JS. Methods of examination, normal anatomy and radiographic findings of chest disease. In: Brant WE, Helms CA, eds. Fundamentals of diagnostic radiology. Philadelphia: Lippincott Williams \& Wilkins, 2007: 360 .

2. Schneider DJ, Moore JW. Patent ductus arteriosus. Circulation 2006; 114:1873-82.

3. Cassidy HD, Cassidy LA, Blackshear JL. Incidental discovery of a patent ductus arteriosus in adults. J Am Board Fam Med 2009; 22:214-8.

4. Pas D, Missault L, Hollanders G, Suys B, De Wolf D. Persistent ductus arteriosus in the adult: clinical features and experience with percutaneous closure. Acta Cardiol 2002; 57:275-8.

5. Goitein O, Fuhrman CR, Lacomis JM. Incidental finding on MDCT of patent ductus arteriosus: use of CT and MRI to assess clinical importance. AJR Am J Roentgenol 2005; 184:1924-31.

6. Zarich S, Leonardi H, Pippin J, Tuthill J, Lewis S. Patent ductus arteriosus in the elderly. Chest 1988; 94:1103-5.

7. Hoffman JI, Kaplan S. The incidence of congenital heart disease. J Am Coll Cardiol 2002; 39:1890-900.

8. Morgan-Hughes GJ, Marshall AJ, Roobottom C. Morphologic assessment of patent ductus arteriosus in adults using retrospectively ECG-gated multidetector CT. AJR Am J Roentgenol 2003; 181:749-54.

9. Fisher RG, Moodie DS, Sterba R, Gill CC. Patent ductus arteriosus in adults-long-term follow-up: nonsurgical versus surgical treatment. J Am Coll Cardiol 1986; 8:280-4.

10. Marquis RM, Miller HC, McCormack RJ, Matthews MB, Kitchin AH. Persistence of ductus arteriosus with left to right shunt in the older patient. Br Heart J 1982; 48:469-84

11. Halpern EJ. Triple-rule-out CT angiography for evaluation of acute chest pain and possible acute coronary syndrome. Radiology 2009; 252:332-45.

12. Takakuwa KM, Halpern EJ. Evaluation of a "triple rule-out" coronary CT angiography protocol: use of 64-Section CT in low-to-moderate risk emergency department patients suspected of having acute coronary syndrome. Radiology 2008; 248:438-46.

13. Leschka S, Oechslin E, Husmann L, et al. Pre- and postoperative evaluation of congenital heart disease in children and adults with 64-section CT. Radiographics 2007; 27:829-46.

14. Kalavrouziotis G, Kourtesis A, Paphitis C, Azariades P. Closure of a large patent ductus arteriosus in children and adults with pulmonary hypertension. Hellenic J Cardiol 2010; 51:15-8. 


\section{SINGAPORE MEDICAL COUNCIL CATEGORY 3B CME PROGRAMME}

\section{(Code SMJ 201409B)}

Question 1. Regarding ductus arteriosus:

(a) It connects the proximal descending thoracic aorta to the roof of the main pulmonary artery or proximal left pulmonary artery.

(b) Right ventricular output is diverted away from the high-resistance fetal pulmonary circulation via the ductus into the descending thoracic aorta.

(c) Structural closure from vasoconstriction, with contraction of the medial smooth muscle in the ductus, occurs within 24-48 hours after delivery.

(d) Persistence of ductal patency at the first three weeks of life is considered pathological.

Question 2. Regarding patent ductus arteriosus (PDA):

(a) Isolated PDA is a common congenital heart anomaly that is estimated to account for approximately $10 \%-12 \%$ of congenital heart anomalies.

(b) The male to female ratio is 1:2 in most reports.

(c) Haemodynamically significant PDA has typically been associated with cardiovascular derangements, including congestive heart failure, arrhythmias and progressively severe pulmonary hypertension.

(d) There is associated risk of infective endarteritis, even in small PDA.

Question 3. Regarding the haemodynamics of PDA:

(a) If the pulmonary vascular resistance exceeds that of the systemic circulation, the flow within the duct reverses to right-to-left.

(b) Eisenmenger's syndrome is an irreversible complication secondary to longstanding elevated pulmonary arterial pressure and associated pulmonary vascular disease.

(c) Most PDAs are haemodynamically well tolerated and remain subclinical into adulthood.

(d) A well-tolerated unrepaired PDA may become clinically significant in conjunction with an acquired physiological insult such as ischaemic heart disease.

Question 4. The following are known imaging features of PDA and pulmonary arterial hypertension:

(a) Chest radiographs may show features of heart failure and pulmonary arterial hypertension.

(b) The extent of calcification of PDA is accurately estimated on chest radiography.

(c) The upper limit of normal for the transverse diameter of the proximal right interlobar artery, as measured on a posterior-anterior radiograph at a level immediately lateral to the proximal portion of the bronchus intermedius, is $17 \mathrm{~mm}$ in men.

(d) Multiplanar computed tomography (CT) reformation provides precise information on the size and morphology of a PDA.

Question 5. The following statements are true:

(a) Triple-rule-out multidetector CT (TRO MDCT) is performed with cardiac gating.

(b) TRO MDCT is most appropriate when acute coronary syndrome, along with other diagnoses such as pulmonary embolism, acute aortic syndrome or nonvascular disease in the thorax, is suspected.

(c) Surgical closure of PDA is recommended when Eisenmenger's syndrome is present.

(d) Nonsurgical transcatheter PDA closure is the treatment of choice whenever possible, as it is less invasive and has less patient morbidity.

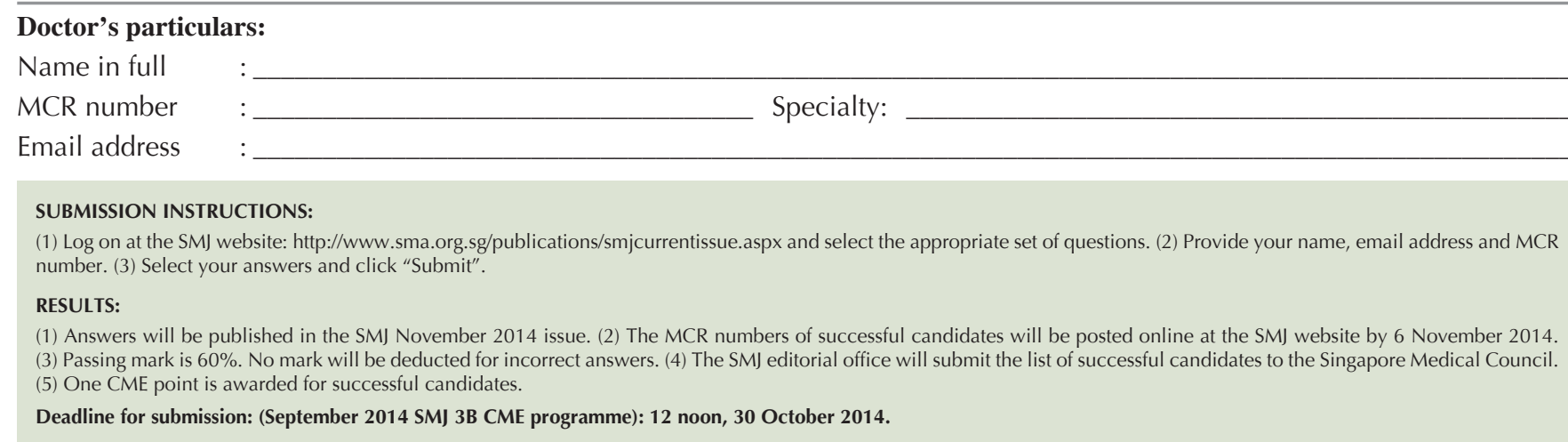

